\title{
Shared Pathogenesis in Depression and Cancer Depicting the Anti-cancer Properties of Anti-depressants
}

\author{
Abhijeet Faye \\ Consultant Psychiatrist, National Cancer Institute, Khasara No 25, Outer Hingna Ring Road, Mauza, Jamtha, Nagpur, Maharashtra, \\ India.
}

\begin{abstract}
Pathogenesis of depression is similar to that of cancer in many aspects. Important mechanisms include effects on hypothalamo-pituitary-adrenal axis (HPA axis), functions of prostaglandins, inflammatory markers and cellular immunity. Anti-depressants must have the actions through above mentioned mechanisms when used to treat depression. Literature has mentioned the effects of various anti-depressant agents in reversing or modifying the pathogenic changes that occur in cancer too. It's worth exploring the anti-cancer properties of anti-depressants and utilize those for the treatment of cancer whenever used for treating associated depression or other psychiatric conditions. Long term studies are needed to prove this less researched aspect of antidepressants.
\end{abstract}

Keywords: Depression- cancer- anti-depressants- anti-cancer properties

Asian Pac J Cancer Biol, 6 (1), 3-4

\section{Introduction}

Depression is common occurrence in cancer patients and antidepressants are widely used to treat depression, pain symptoms, chronic fatigue and anxiety among many other indications in cancer patients. Depression is associated with stress related chronic inflammatory changes and effects on hypothalamo-pituitary adrenal axis (HPA axis) in the body besides alteration in the levels and functioning of neurotransmitters like serotonin, nor-epinephrine \& dopamine. Anti-depressants are known to act through all these mechanisms. There are many such molecular, pathological or immunological reactions that occur both in cancer too.

\section{HPA axis \& stress}

Depression and stress are positively correlated. Depression is either caused or precipitated by the stress of any kind. The chronic activation of the HPA axis in depression impairs the immune response and may contribute to the development and progression of certain cancers (e.g breast \& prostate cancer). Studies have described the instances where the onset of cancer has been first recognized immediately following some stressor, a financial crisis, a disaster, bereavement or an accident.
Submission Date: 08/21/2020Ａcceptance Date: 01/14/2021

\section{Prostaglandins}

Prostaglandins are the ephemeral signallers self-regulating every cell in the body, including those serving for mood and immunity. Depression predisposes an individual to infection, osteoporosis, cancer, cardio-vascular diseases, neurodegenerative and auto-immune disorders and prostaglandins are incriminated in this causation. Excessive prostaglandin synthesis may depress immune function and induce cancer [1]. Similarly, prostaglandins are involved in various processes of carcinogenesis like signal disruption, cyclo-oxygenase up regulation, synthesis \& expression of oncogene, increased cell replication, viral activation, changes in apoptosis, tumour initiation \& promotion, metastasis, angiogenesis, immune-suppression and activation of mitochondria. Research mentions that chronic use of prostaglandin inhibitors like aspirin or ibuprofen reduced the risk of colon cancer by around $40 \%$. Prostaglandins and thromboxanes are also involved in metastasis by neovascularisation and proteolytic enzyme production.

\footnotetext{
Corresponding Author:

Dr. Abhijeet Faye

Consultant Psychiatrist, National Cancer Institute, Khasara No 25, Outer Hingna Ring Road, Mauza, Jamtha, Nagpur, Maharashtra, India.

Email: abhijeetfaye12@gmail.com
} 


\section{Inflammatory markers}

Recently, the role of neuro-inflammation has been found in the pathogenesis of depression especially that of NLRP3-inflammasome complex [2]. Studies have mentioned the role of inflammasomes or their products, mainly IL $1 \beta$ and IL-18 on carcinogenesis and progression of tumor also. These inflammasomes can be the therapeutic targets in cancer.

\section{Cellular immunity}

Depression is associated with the decreased activity of cytotoxic T cell and natural killer cells which affects the immune surveillance of tumours, occurrence \& accumulation of somatic mutations and genomic instability [3]. Thus immune-suppression can be the aetiology or the consequence of the cancer and same is true for depression.

\section{Antidepressants}

Studies showed that antidepressants have potent anti-neoplastic properties, both in vitro and in vivo. They are found to destroy the cells or arrest their proliferation [4]. Antidepressants keep a check on prostaglandin-synthesizing enzymes, they are cytotoxic and cytostatic (convert multidrug resistant cells to sensitive, and protect non-malignant cells from radiation and chemotherapy).

Antidepressant like fluoxetine, paroxetine, amitriptyline, mirtazapine, desvenlafaxine, venlafaxine, mianserin, imipramine and agomelatine are found to inhibit inflammasome by decreasing the serum levels of IL-1 $\beta$ \& IL-1 8 and by reducing the NLRP3 \& IL-1 $\beta$ (p17) protein expression [2]. They also have anti-inflammatory action on IL- 6 and TNF- $\alpha$. Research evidence shows that sertraline has an anti-tumor activity noticed by its effect on the cell viability and cell proliferation. Similarly, by inducing the apoptosis in myeloid leukemia HL-60 cells clomipramine, imipramine and citalopram were found to have anti-cancer properties. Paroxetine was also found to induce the apoptosis in human osteosarcoma cells. The effect was associated with p38 MAPK and caspase-3 pathways activation [5].

Thus, there is a sharing of pathogenesis' mechanisms by depression \& cancer and anti-cancer properties of antidepressants can be utilized for the treatment of cancer whenever used for treating associated depression or other psychiatric conditions. Long term studies are needed to prove this less researched aspect of antidepressants.

\section{References}

1. Lieb J. Defeating Cancer With Antidepressants. ecancermedicalscience. 2008;. https://doi.org/10.3332/ ecancer.2008.88

2. Alcocer-Gómez E, Casas-Barquero N, Williams MR, RomeroGuillena SL, Cañadas-Lozano D, Bullón P, Sánchez-Alcazar JA, Navarro-Pando JM, Cordero MD. Antidepressants induce autophagy dependent-NLRP3-inflammasome inhibition in Major depressive disorder. Pharmacological Research. 2017 07;121:114-121. https://doi.org/10.1016/j. phrs.2017.04.028
3. Reiche EMV, Nunes SOV, Morimoto HK. Stress, depression, the immune system, and cancer. The Lancet Oncology. 2004 Oct;5(10):617-625. https://doi.org/10.1016/s14702045(04)01597-9

4. Arimochi H, Morita K. Characterization of cytotoxic actions of tricyclic antidepressants on human HT29 colon carcinoma cells. European Journal of Pharmacology. 2006 07;541(12):17-23. https://doi.org/10.1016/j.ejphar.2006.04.053

5. Chou C, He S, Jan C. Paroxetine-induced apoptosis in human osteosarcoma cells: Activation of p38 MAP kinase and caspase- 3 pathways without involvement of $[\mathrm{Ca} 2+]$ i elevation. Toxicology and Applied Pharmacology. 2007 02;218(3):265-273. https://doi.org/10.1016/j. taap.2006.11.012

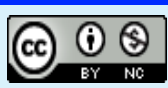

This work is licensed under a Creative Commons AttributionNon Commercial 4.0 International License. 